\title{
O USO DE VEÍCULOS AÉREOS NÃO TRIPULADOS (VANT) PARA GERAÇÃO DE DADOS GEOCARTOGRÁFICOS NA UNIVERSIDADE DE PERNAMBUCO - CAMPUS GARANHUNS
}

\author{
Rodolfo Alexandre da Silva Gomes de Deus ${ }^{(a)}$, Renilson Pinto da Silva Ramos ${ }^{(b)}$, Fernando da \\ Silva Alexandre ${ }^{(\mathrm{c})}$, Daniel Dantas Moreira Gomes ${ }^{(\mathrm{d})}$ \\ (a)Universidade de Pernambuco - UPE/Campus Garanhuns, rdolfodeus@gmail.com \\ (b)Universidade de Pernambuco - UPE/Campus Garanhuns, renilsonr5 @ hotmail.com \\ (c)Universidade de Pernambuco - UPE/Campus Garanhuns, fnando257@gmail.com; \\ (d)Universidade de Pernambuco - UPE/Campus Garanhuns, daniel.gomes@upe.br
}

\section{RESUMO}

\begin{abstract}
O presente trabalho tem como objetivo utilizar um veículo aéreo não tripulado para gerar produtos geocartográficos. A utilização de VANT na obtenção de imagens para geração de produtos geocartográficos, acaba proporcionando uma rápida aquisição de dados a nível local, e permite que tais dados sirvam de base para futuros planejamentos e tomadas de decisões por parte do poder administrativo local. Outra vantagem é a possibilidade de revisitação da área em curtos períodos de tempo. A área de entorno da Universidade de Pernambuco foi escolhida por se tratar de uma área pequena, visto que a aeronave utilizada foi um quadcóptero multirotor que não possui bom desenvolvimento para trabalhar em áreas grandes, na fomentação dos dados utilizou-se das imagens obtidas através do VANT Phantom 3 Advanced da empresa DJI. Todos os dados foram utilizados objetivando extrair o maior número possível de informações para melhor conhecimento da área.
\end{abstract}

Palavras-chave: multirotor, quadcóptero, monitoramento, contribuição.

\section{Introdução}

O uso de veículos aéreos não tripulados (VANT) tem se tornado algo bastante comum nos dias atuais, por permitirem uma aquisição mais rápida de dados locais, os mesmos acabaram por se consolidar como ferramentas essenciais e de uso indispensável em diversos setores, seja na mineração, na agricultura de precisão, no cadastramento de imóveis rurais, dentre outros. De acordo com Silva (2015) o interesse no uso de VANT no Brasil tem se intensificado em diversas aplicações nos últimos anos. Ainda segundo o autor, o número de usuários atraídos a essa tecnologia aumenta exponencialmente a cada dia, e esses usuários estão em diversos campos de atividade, seja privado, governamental, militar ou até mesmo científico.

Segundo Jorge (2014) o termo "Veículo Aéreo Não Tripulado" é mundialmente reconhecido e inclui uma grande gama de aeronaves que são autônomas, semiautônomas ou remotamente operadas. A associação Brasileira de Aeromodelismo define VANT como: "um veículo capaz de voar na atmosfera, fora do efeito de solo, que foi projetado ou modificado para não receber um piloto humano e que é operado por controle remoto ou autônomo". 
XVII Simpósio Brasileiro

de Geografia Fisica Aplicada

I Congresso Nacional

de Geografia Física

\section{OS DESAFIOS DA GEOGRAFIA FÍSICA NA FRONTEIRA DO CONHECIMENTO \\ Instituto de Geociências - Unicamp \\ Campinas - SP \\ 28 de Junho à 02 de Julho de 2017}

Os produtos gerados através de VANT possuem em sua constituição dados geocartográficos que permitem através do geoprocessamento dos mesmos a fomentação de diversos mapas, índices e análises, como por exemplo realizar medições, calcular volume de pilhas de descarte ou lixões, criar ortofotos e ortomosaicos da área escolhida, criar modelos digitais de superfície e de terreno, além de possibilitar a criação de modelos tridimensionais dos alvos de estudo, permitindo dessa forma transportar para o escritório ou laboratório um modelo 3D da área real, permitindo análises de diversos tipos e conhecimento geral do objeto sem necessidade de ir a campo para além do voo de obtenção das imagens. Este artigo tem por finalidade utilizar imagens aéreas de um VANT para gerar produtos geocartográficos de uma área pequena, apenas para visualização do potencial de uso de imagens aerofotogramétricas em campo científico. Para isso foi escolhido a área de entorno da Universidade de Pernambuco Campus Garanhuns local de origem dos autores, para criação dos dados aqui representados foi utilizado o software Agisoft Photoscan Professional em todos os processos de processamento digital de imagens.

\subsection{Localização do objeto de estudo}

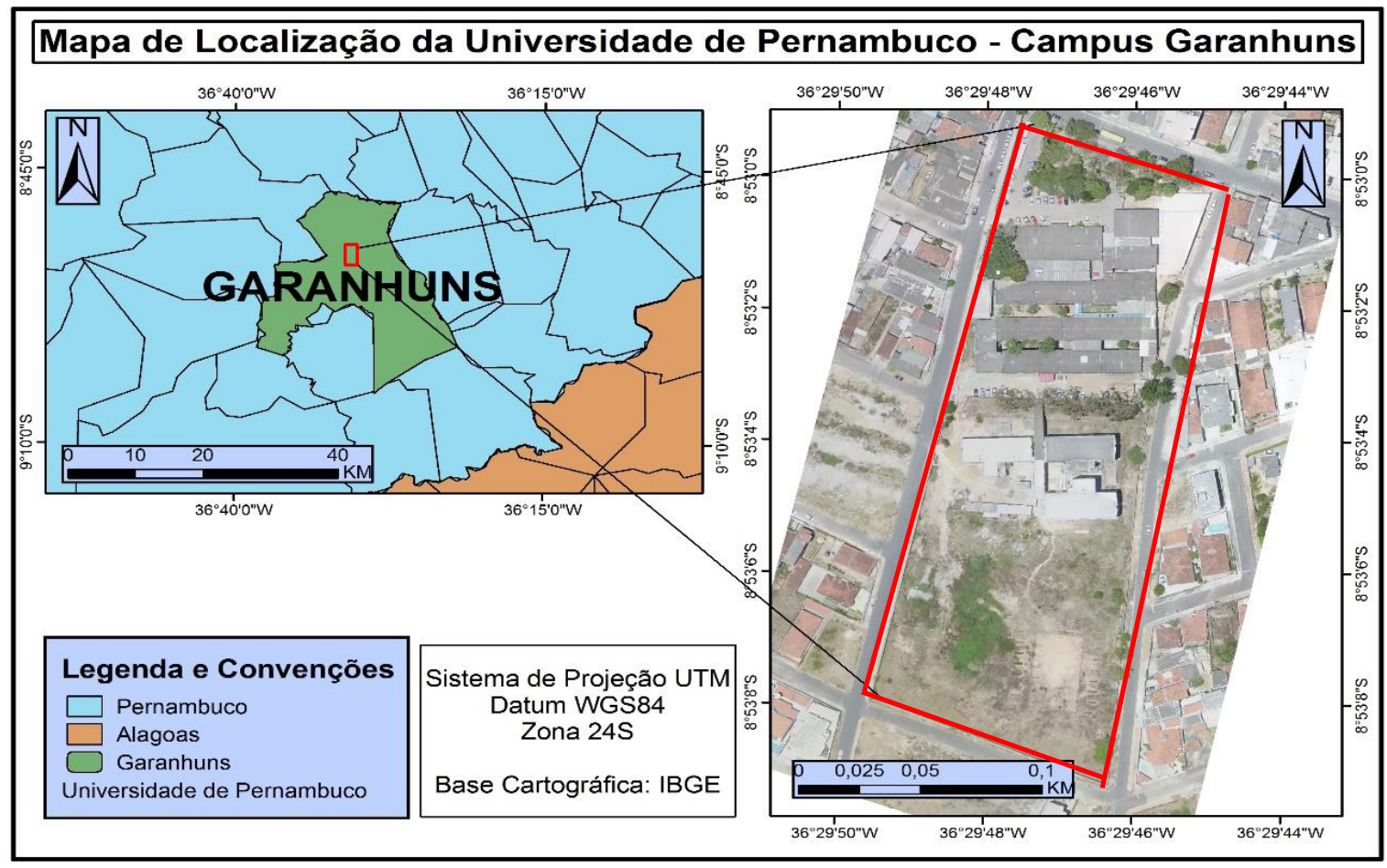

Figura 01: Mapa de Localização da Universidade de Pernambuco.

Fonte: Desenvolvido pelos autores. 
XVII Simpósio Brasileiro

de Geografia Fisica Aplicada

I Congresso Nacional

de Geografia Física

\section{OS DESAFIOS DA GEOGRAFIA FÍSICA NA FRONTEIRA DO CONHECIMENTO \\ Instituto de Geociências - Unicamp \\ Campinas - SP \\ 28 de Junho à 02 de Julho de 2017}

\section{Metodologia de Trabalho}

\subsection{Materiais}

Objetivando uma configuração mais otimizada na visualização das etapas que compõem a metodologia utilizada, um fluxograma foi criado para dispor em sequência todo o passo a passo da realização do trabalho, desta forma, os processos feitos e atividades realizadas são melhor observadas quando dispostos nesse tipo de recurso visual.

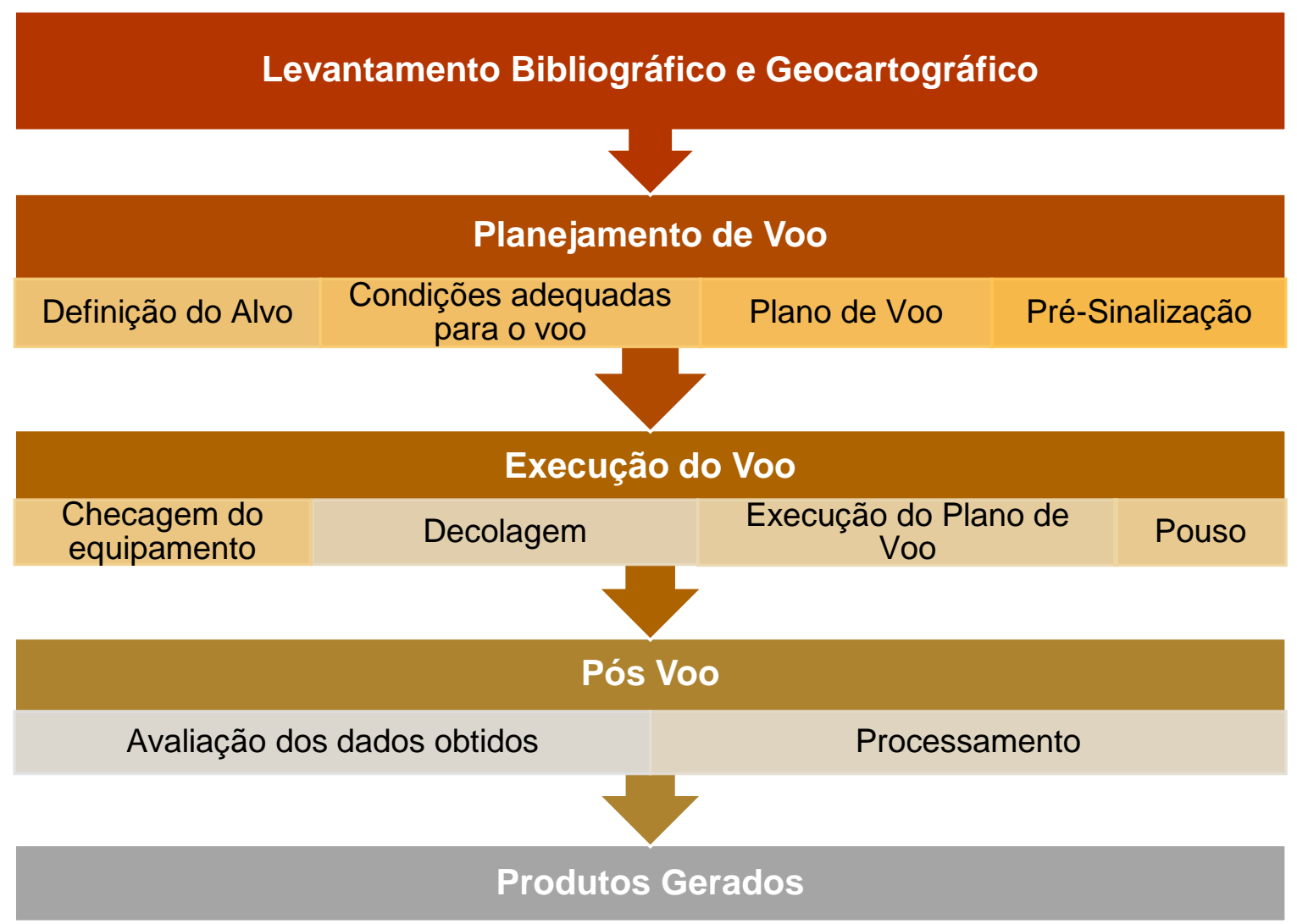

Figura 02: Fluxograma Metodológico.

Fonte: Desenvolvido pelos autores

A primeira etapa do trabalho consiste no levantamento e aquisição de material bibliográfico referente à área de estudo que possa oferecer embasamento teórico para a fomentação da pesquisa. Foram consultados artigos, monografias, livros, dissertações, teses, entre outros materiais, para dar suporte teórico a pesquisa e empregar os dados geocartográficos obtidos de forma a contribuir com o arcabouço teórico da ciência para o objeto de estudo. 


\subsection{Planejamento de voo}

A segunda etapa consiste no planejamento de voo, nesta etapa o alvo foi definido, e após a definição do alvo uma análise prévia das condições para o voo foi realizada, buscando saber a respeito das condições meteorológicas, após uma rápida verificação da possibilidade de voo no dia em questão, o plano de voo foi criado tendo por base as considerações de Fitz (2008) que fala que é necessário haver no mínimo uma sobreposição longitudinal de $60 \%$ e lateral de 30\%, desta forma e visto que se tratava de uma área pequena foi decidido que o voo teria recobrimento longitudinal de $80 \%$ e lateral de $60 \%$.

\subsection{Execução do voo}

A terceira etapa teve por finalidade executar o voo, antes da execução propriamente dita, uma verificação do equipamento em solo foi realizada visando notar qualquer tipo de problema que pudesse gerar um problema na missão que poderia vir a ser abortada mediante esses problemas não resolvidos previamente. O VANT utilizado foi um Phantom 3 Advanced da empresa DJI, por se tratar de um quadcóptero multirotor, ele não necessita de nenhum tipo de assistência no lançamento para o voo, podendo decolar verticalmente de um ponto qualquer no terreno que não ofereça nenhum perigo para a execução desse passo, em modo ainda manual o VANT foi elevado até os 50 metros de altitude para só assim dar início ao plano de voo previamente realizado que por sua vez elevou a aeronave até os 100 metros de altitude e iniciou o voo pré-programado realizando a tomada de cenas, todo o processo não chegou a durar 10 minutos, pois por se tratar de um multirotor sua bateria suporta em torno de 23 minutos de voo, e também por se tratar de uma área pequena como já falado anteriormente não mais do que 10 minutos foram necessários para realização do voo por completo. Após a conclusão do voo préprogramado a aeronave volta ao ponto de partida por meio dos GPS's que possui embarcados e através do comando Go to home, onde pousou de forma vertical em um raio de 3 metros do ponto de decolagem.

\subsection{Pós voo}

$\mathrm{Na}$ quarta etapa de pós voo, ocorreu a avaliação das cenas tomadas na etapa anterior, detalhes como a rota da aeronave no ar o contraste e a nitidez das imagens são verificados, objetivando uma boa qualidade de imagens para processamento, e a verificação do caminho realizado pela aeronave buscando observar se a mesma não se distanciou muito do projeto inicial sendo levada por elementos naturais como o vento, ou mesmo por falhas no plano estabelecido. Após essas verificações iniciais pós voo todos os dados são armazenados em um Geodatabase no ArcGis 10.2.2, para processamento posterior por meio do software Agisoft Photoscan Professional.

No ambiente do Photoscan foram gerados nuvem de pontos, ortomosaico, modelo digital de elevação e modelo tridimensional da área em estudo. Através de cinco etapas base o Photoscan realiza o 


\section{OS DESAFIOS DA GEOGRAFIA FÍSICA NA FRONTEIRA DO CONHECIMENTO \\ Instituto de Geociências - Unicamp \\ Campinas - SP \\ 28 de Junho à 02 de Julho de 2017}

processamento, são elas: 1 - calibração automática da câmera, que tem por base os dados contidos no

EXIF das fotografias; 2 - alinhamento das fotos, onde ele usa os pontos que encontra em comum nas fotografias; 3 - geração da nuvem de pontos, a partir de um estimativo de posição das cenas tomadas, são identificados as coordenadas x, y e z; 4 - criação de um MDS de malha triangular, onde a nuvem de pontos é utilizada como nós, e a partir daí gera-se uma estrutura vetorial com topologia do tipo nó-arco que representa a superfície através de faces triangulares que se interligam; 5 - geração do ortomosaico, que é criado a partir da texturização da geometria construída pela malha triangular gerada no passo anterior.

\section{Resultados e Discussão}

O VANT utilizado o Phantom 3 Advanced por se tratar de um multirotor não é recomendado para cobrir grandes áreas, mas numa pequena área como a escolhida o mesmo desempenhou um ótimo papel, possibilitando a obtenção de cenas para processamento posterior e assim geração de diversos produtos que serão aqui apresentados.

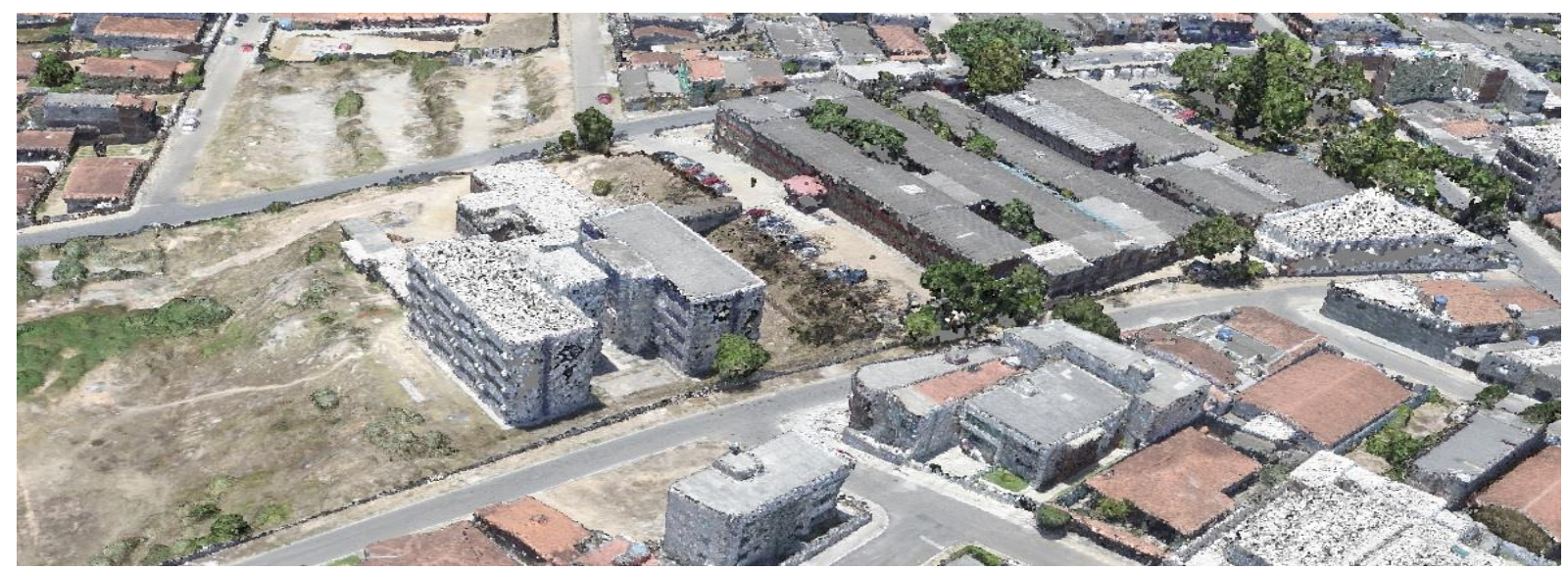

Figura 03: Nuvem de Pontos.

Fonte: Desenvolvido pelos autores.

Após a calibração da câmera e o alinhamento das fotos que é realizado tendo por base os pontos comuns entre as fotografias, é gerada a nuvem de pontos que servirá de base para todos os processamentos posteriores, por se tratar de uma evolução dos pontos comuns ela apresenta uma característica bem mais completa que os pontos homólogos, a partir das posições das fotografias pré-estabelecidas nos passos anteriores, na nuvem de pontos são identificadas as coordenadas x, y e z. 


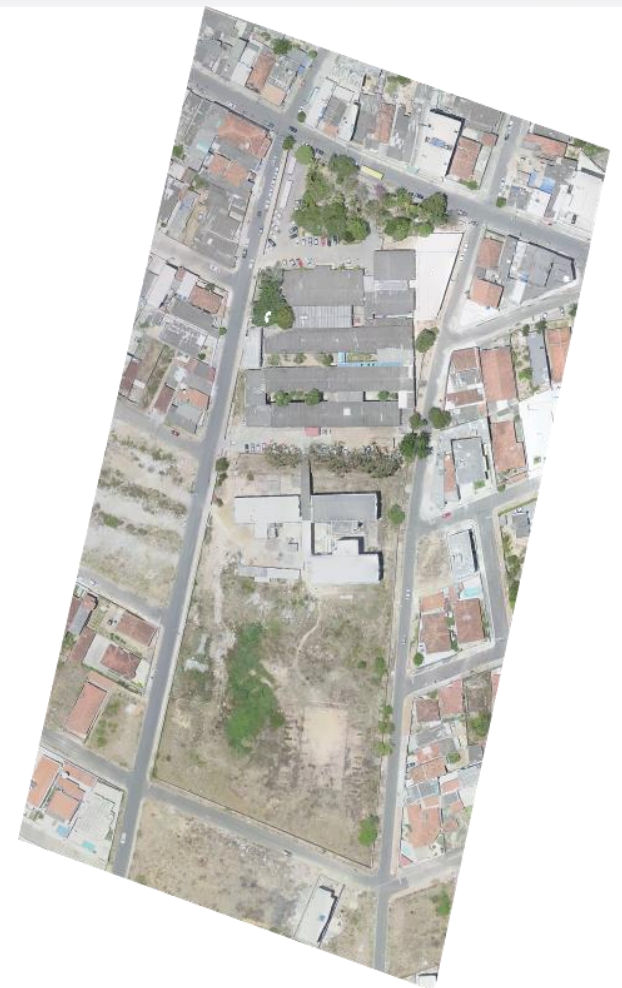

Figura 04: Ortomosaico.

Fonte: Desenvolvido pelos autores.

Quando o modelo digital de superfície é criado, ele gera uma malha triangular que utiliza a nuvem de pontos como nós que acaba por criar uma estrutura do tipo vetorial que possui uma topologia do tipo nó-arco que busca representar a superfície da área escolhida através de um conjunto de faces triangulares interligadas, então a partir da texturização dessa geometria é criado o ortomosaico.

O modelo digital de elevação demonstra através de cotas altimétricas o nível de variação de elevação da área escolhida, a partir de um esquema de cores, onde as cores mais quentes representam os pontos mais altos do terreno e as cores mais frias os pontos mais baixos ele representa essa variação através desse recurso visual de forma bastante edificante. 


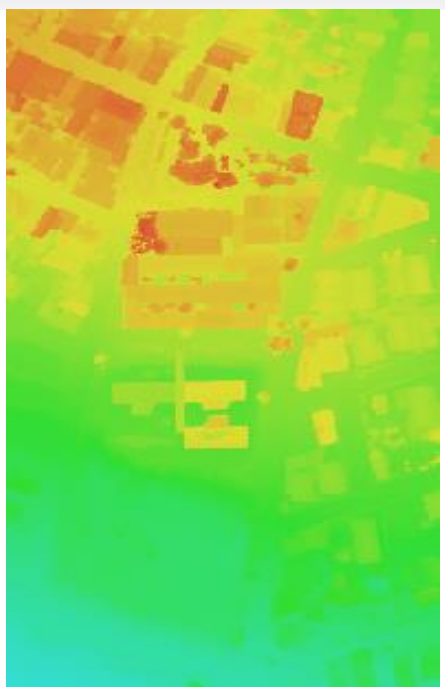

Figura 05: Modelo Digital de Elevação.

Fonte: Desenvolvido pelos autores.

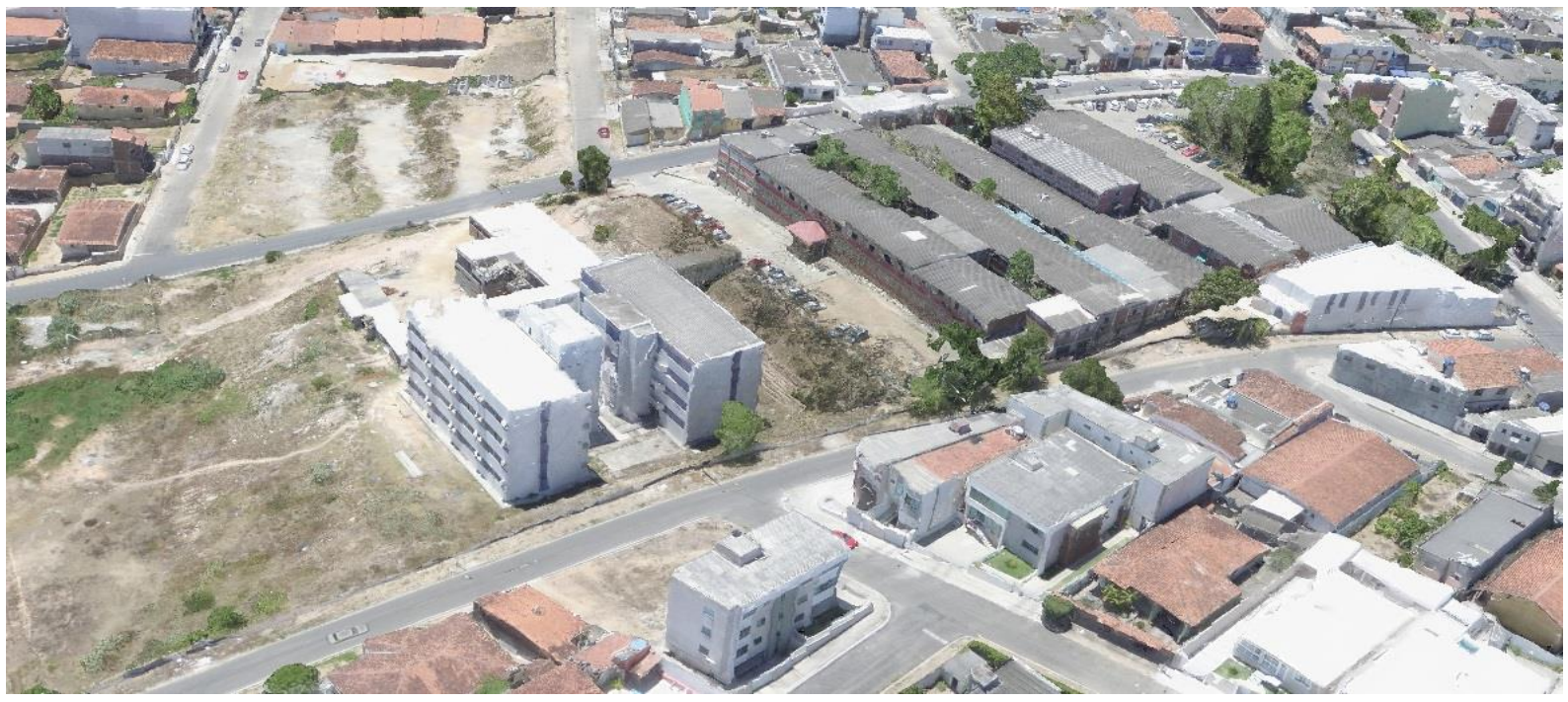

Figura 06: Modelo Tridimensional

Fonte: Desenvolvido pelos autores.

Por fim o modelo tridimensional da área que foi gerado em alta qualidade o que acarretou um processamento de 20 horas representa a área de estudo em todas suas formas e cores as mais semelhantes possíveis com a realidade. Esse modelo tridimensional gerado por veículos aéreos não tripulados é algo de imensurável valor, pois transporta para o ambiente computacional a realidade do terreno, onde, por exemplo, em plantações é possível fazer com que o profissional do campo como um agrônomo ou técnico agrícola possa observar a situação da plantação sem necessariamente ir a campo, economizando, portanto, tempo e mão de obra. É possível realizar análises prévias do terreno em cima desse modelo 3D para que assim decisões possam ser tomadas e campos de ação possam ser escolhidos sem a necessidade de fazer um reconhecimento local, todas essas possibilidades geram economia de capital e 
trazem para dentro do escritório, gabinete ou laboratório informações acerca do alvo escolhido de forma prática, rápida e que pode ser obtida em poucos dias de processamento.

\section{Conclusões}

O uso de um veículo aéreo não tripulado no trabalho aqui realizado acabou por se mostrar uma ferramenta de bastante eficácia na fomentação de produtos geocartográficos, apesar da aeronave utilizada ser de pequeno porte e cobrir apenas pequenas áreas, ela desempenhou um papel deveras importante e cumpriu com tudo aquilo que foi previsto para o trabalho.

Todos os produtos gerados servem como suporte geocartográfico para criação de diversas análises, como por exemplo através do ortomosaico ser possível observar as caixas d'água locais que estiverem destampadas para assim facilitar o trabalho de agentes de endemias do município que podem se guiar pelo ortomosaico para agir diretamente em focos de dengues dentre outras endemias. Através do mesmo produto é possível notar delimitar uma malha de arruamentos e criar um mapa a nível local de todas as ruas da área abrangida. Com o DEM é possível notar o nível de elevação da área de estudo, facilitando, portanto, a ação do governo municipal para tomada de decisões e implementação de medidas diversas por exemplo.

\section{Agradecimentos}

1. Os autores agradecem a Universidade de Pernambuco (UPE) pelo financiamento dos recursos do projeto de pesquisa "Análise de riscos socioambientais em bacias hidrográficas através da utilização de ferramentas de geoprocessamento e aerofotogrametria de pequeno formato", junto ao Programa de Fortalecimento Acadêmico (PFA/IC), e ao Laboratório de Geoprocessamento e Modelagem Ambiental pelo apoio em todo o decorrer do trabalho.

\section{Referências Bibliográficas}

BERNARDI, A. C. de C.; NAIME, J. de M.; RESENDE, A. V. de; BASSOI, L. H.; INAMASU, R. Y. Agricultura de precisão: resultados de um novo olhar. Brasília, DF: Embrapa, 2014.

DUARTE. P.A. Fundamentos de Cartografia. 3. Ed. Florianópolis. UFSC, 2008.

FITZ, P. R. Geoprocessamento sem Complicação. São Paulo, Oficina de Textos, 2008.

FLOREnZANO, T, G. Geomorfologia, Conceitos e técnicas atuais. São Paulo, Oficina de Textos, 2008. 
MITISHITA, E. A.; GONCALVES, J. E; GRACA, N. L. S. S; CENTENO, Jorge Antonio Silva ; MACHADO, Álvaro Muriel Lima . O USO DE VEÍCULOS AÉREOS NÃO TRIPULADOS (VANTS) EM APLICAÇÕES DE MAPEAMENTO AEROFOTOGRAMÉTRICO. In: XXVI Congresso Brasileiro de Cartografia, 2014, Gramado. Anais do XXVI Congresso Brasileiro de Cartografia, 2014.

ROSA. R. Introdução ao sensoriamento remoto. 7 ed. São Paulo: UDUFU. 2009

ROSS, J, S. Geomorfologia: Ambiente e Planejamento. São Paulo: Contexto, 1990.

SILVA, C. A.; DUARTE. C. R.; SOUTO. M. V. S.; SABADIA. J. A. B.: Utilização de VANT para geração de ortomosaicos e aplicação do Padrão de Exatidão Cartográfica (PEC). Anais XVII Simpósio Brasileiro de Sensoriamento Remoto - SBSR, João Pessoa-PB, Brasil, 25 a 29 de abril de 2015, INPE.

SILVA, Cristiano Alves da. DUARTE, Cynthia Romariz. SOUTO, Michael Vandesteen Silva. SANTOS, André Luis Silva Dos. AMARO, Venerando Eustaquio. BICHO, Cristina Prando. SABADIA, José Antonio Beltrão. Avaliação da acurácia do cálculo de volume de pilhas de rejeito utilizando VANT, GNSS e LiDAR. BCG Boletim de Ciências Geodésicas - On-Line version, ISSN 1982-2170.

SILVA, Cristiano Alves da. SOUTO, Michael Vandesteen Silva. DUARTE, Cynthia Romariz. BICHO, Cristina Prando. SABADIA, José Antonio Beltrão. Avaliação da acurácia dos ortomosaicos e modelos digitais do terreno gerados pelo MVANT/DNPM. Revista Brasileira de Cartografia (2015) N0 67/7: 1479-1495.

SILVA, Wagner Fernando. SILVA, Lenildo Santos. MALTA, Édio Albertin. GONDIM, Rodolpho de Oliveira.

WARREN, Morris Scherer. Avaliação de uso de Veículo Aéreo Não Tripulado - VANT em atividades de fiscalização da Agência Nacional de Águas. Anais XVII Simpósio Brasileiro de Sensoriamento Remoto - SBSR, João Pessoa-PB, Brasil, 25 a 29 de abril de 2015, INPE. 